\title{
Verdient - Unverdient. Der öffentliche Diskurs um die Erbschaftssteuer in Deutschland und Österreich
}

\author{
Jens Beckert • H. Lukas R. Arndt
}

\section{MPIfG Journal Article}

Jens Beckert, H. Lukas R. Arndt: Verdient - Unverdient: Der öffentliche Diskurs um die Erbschaftssteuer in Deutschland und Österreich. In: Berliner Journal für Soziologie 27(2), 271-291 (2017). Springer VS

The original publication is available at the publisher's web site: https://doi.org/10.1007/s11609-017-0346-2

The MPIfG Journal Articles series features articles by MPIfG researchers and visiting scholars published in peer-reviewed journals. Max Planck Institute for the Study of Societies (MPIfG) Cologne | www.mpifg.de

Online publiziert: 19. Dezember 2017

(C) Springer Fachmedien Wiesbaden GmbH, ein Teil von Springer Nature 2017

Zusammenfassung Trotz des erheblichen Einflusses der Vermögensvererbung auf die generationsübergreifende Fortschreibung sozialer Ungleichheit spricht sich sowohl in Deutschland als auch in Österreich eine Mehrheit der Bevölkerung gegen die Erbschaftssteuer aus. In diesem Beitrag untersuchen wir, wie Gegner und Befürworter der Steuer ihre jeweilige Position begründen, und ergründen so die Strukturen des Diskurses zur Erbschaftssteuer. Datengrundlage sind zwei Online-Diskussionen, die jeweils durch ausführliche Interviews zu dem Thema ausgelöst wurden. Insgesamt wurden 3573 Argumente inhaltsanalytisch codiert und ausgewertet. Die Ergebnisse bestätigen den Befund einer überwiegenden Ablehnung der Erbschaftssteuer. Die Auswertung der Positionsbegründungen gibt darüber hinaus jedoch Einblicke in die vielschichtigen Kontroversen, die sich mit der normativen und funktionalen Problematik dieser Steuer verbinden.

Schlüsselwörter Erbschaftssteuer · Vermögensvererbung · Finanzsoziologie · Inhaltsanalyse $\cdot$ Österreich · Deutschland · Online-Diskussion · Soziale Ungleichheit

\section{Deserved - undeserved. The public discourse on inheritance tax in Germany and Austria}

\begin{abstract}
Despite the significant impact of inheritance on perpetuating social inequality from one generation to the next, a majority of Germans and Austrians are opposed to an inheritance tax. In the present contribution, we investigate how opponents and proponents of such a tax justify their respective positions in order to understand the structure of the discourse on this issue. The paper draws on our
\end{abstract}

\footnotetext{
J. Beckert $(\bowtie) \cdot$ H. L. R. Arndt $(\bowtie)$

Max-Planck-Institut für Gesellschaftsforschung, Paulstr. 3, 50676 Köln, Deutschland

E-Mail: Beckert@mpifg.de; arndt@mpifg.de
} 
content analysis of 3573 arguments, expressed in two online debates that were both triggered by in-depth interviews on the topic. The results confirm that a majority rejects an inheritance tax. Yet beyond this finding, the analysis of the respective justifications offers insights into the complex controversies this tax and the normative and functional questions it poses give rise to.

Keywords Inheritance tax $\cdot$ Inheritance $\cdot$ Fiscal sociology $\cdot$ Content analysis · Austria $\cdot$ Germany $\cdot$ Online discussion $\cdot$ Social inequality

\section{Mérité ou immérité? Le discours public sur les droits de succession en Allemagne et en Autriche}

Résumé En dépit de l'influence considérable de la transmission du patrimoine sur la reproduction des inégalités sociales entre générations, une majorité de la population allemande et autrichienne est opposée aux droits de succession. Cet article étudie la manière dont partisans et opposants aux droits de succession justifient leur position et examine ainsi les structures du débat sur la question. Il s'appuie sur une analyse du contenu de 3573 arguments exprimés sur deux forums de discussion en ligne qui ont été choisis à la suite d'entretiens approfondis sur le sujet. Les résultats obtenus confirment la thèse d'un rejet majoritaire des droits de succession. L'analyse des justifications proposées donne en outre un aperçu de la complexité des débats sur les problèmes normatifs et fonctionnels liés aux droits de succession.

Mots-clés Droits de succession - Transmission du patrimoine - Sociologie de la finance $\cdot$ Analyse de contenu A Autriche - Allemagne - Discussion en ligne · Inégalité sociale

\section{Einleitung}

Simulationsrechnungen beziffern das jährlich in Deutschland vererbte Vermögen auf bis zu 400 Milliarden Euro (Tiefensee und Grabka 2017). Für Österreich zeigen auf Befragungen basierende Untersuchungen ein derzeitiges Erbschaftsvolumen von knapp über 10 Milliarden Euro (Altzinger und Humer 2013, S. 22). Dieses Volumen dürfte jedoch weit höher liegen, da Befragungen das tatsächliche Erbvolumen erheblich unterschätzen ${ }^{1}$.

In beiden Ländern ranken sich um den Transfer von Vermögen zwischen den Generationen erhebliche gesellschaftspolitische Kontroversen. Diese Kontroversen flackern insbesondere bei Debatten um Reformen der Erbschaftsbesteuerung immer wieder auf. Sowohl in Deutschland als auch in Österreich wurden solche Reformen während der letzten Jahre intensiv diskutiert (Beckert 2013; Gaisbauer et al. 2013).

Für eine Antwort auf die Frage, weshalb Vermögensvererbung eine so kontroverse Institution ist, gibt es etliche Anhaltspunkte. Einerseits ist Vermögensvererbung ein zentrales Instrument der generationsübergreifenden Perpetuierung sozialer Un-

\footnotetext{
1 Siehe auch Altzinger und Humer (2013, S. 81).
} 
gleichheit und steht als „unverdientes Vermögen“ quer zur Legitimation sozialer Ungleichheit aus Leistungsunterschieden. Andererseits wird die Vererbung von Vermögen als genuiner Teil liberaler Eigentumsrechte und des Ausdrucks familiärer Kontinuität und Solidarität gesehen (ausführlich dazu: Beckert 2004, 2013).

Verschiedene Umfragen zur Erbschaftsbesteuerung zeigen, dass sich in Deutschland etwa zwei Drittel der Bevölkerung zumindest skeptisch gegenüber der Erbschaftssteuer zeigen. So waren in einer Infratest-Umfrage von Mai $201365 \%$ der Befragten gegen eine Erhöhung der Erbschaftssteuer, wohingegen sich $53 \%$ für eine Erhöhung der Einkommenssteuer aussprachen (Infratest 2013, S. 4). Eine repräsentative Umfrage des Meinungsforschungsinstituts YouGov ergab 2015, dass in Deutschland eine Mehrheit von $70 \%$ die Besteuerung von Erbschaften grundsätzlich unfair findet und nur $21 \%$ grundsätzlich fair (Schmidt 2015). Für Österreich wurde 2014 die Stimmung durch das Market Institut erhoben. Demnach sprechen sich 39 $\%$ eher für die Wiedereinführung einer „Erbschaftssteuer für alle“ aus und $49 \%$ dagegen. Bei einem Freibetrag von 250.000 Euro hingegen steigt die Zustimmung auf $56 \%$ und nur noch $30 \%$ sprechen sich dagegen aus (Market Institut 2014).

Wie aber begründen Akteure in Deutschland und in Österreich ihre Unterstützung oder Ablehnung der Erbschaftssteuer? Welche Argumente dominieren den öffentlichen Diskurs zu dem Thema? Die existierenden Umfragen zu Einstellungen zur Erbschaftssteuer geben keinerlei Hinweise darauf, wie die Befragten ihre Position begründen. ${ }^{2}$

Zur Untersuchung dieser Frage haben wir die Kommentare in einem deutschen und einem österreichischen Onlineforum ausgewertet, in denen jeweils ein ausführliches Interview zu dem Thema Anlass zu einer Diskussion gab. Die beiden Diskussionen sind insofern vergleichbar, als die in den Interviews vorgebrachten Argumente, trotz einiger Unterschiede, insgesamt eine hohe Übereinstimmung aufweisen. In den Foren äußern sich Personen, denen die Lektüre des jeweiligen Gesprächs genügend Anlass gegeben hat, eine eigene Meinung zu dem Thema zu äußern oder einen Beitrag zu einem Sachverhalt innerhalb der Materie zu verfassen und in dem Forum zu veröffentlichen. Dabei lösen sie sich auch von den im Interview vorgebrachten Argumenten und führen so eine relativ eigenständige Diskussion zum Thema der Erbschaftsbesteuerung. Die Gruppe der Kommentatoren ist dabei nicht repräsentativ für die Gesamtbevölkerung (vgl. Abschnitt 2). Dennoch lassen vorliegende Untersuchungen vermuten, dass öffentliche Online-Diskurse, die von gut informierten Teilnehmern geprägt sind, hinsichtlich der Verteilung der Meinungen mit dem Diskurs der Gesamtbevölkerung vergleichbar sind: „Meinungen, die im Internet häufiger

\footnotetext{
2 Solche Begründungen ließen sich natürlich mithilfe darauf zielender Fragen mit dem Instrument der Umfrageforschung eruieren. Damit könnten repräsentative Daten erlangt werden. Doch haben Umfragen auch zumindest drei erhebliche Nachteile. Erstens wird durch die Standardisierung von Antworten das Ergebnis gelenkt und möglicherweise eingeengt. Zweitens laufen die Ergebnisse Gefahr, durch Effekte der sozialen Erwünschtheit bestimmter Antworten beeinflusst zu sein. Und drittens würden sich in Umfragen auch Personen zu dem Thema äußern, die sich nie oder selten Gedanken um die Erbschaftssteuer machen. Diese Begründungen von Einstellungen zur Erbschaftssteuer würden gleichberechtigt mit Begründungen von Personen eingehen, die sich mit der Frage der Erbschaftssteuer aus eigenem Antrieb beschäftigen und somit auch eher als Wortführer im öffentlichen Diskurs in Erscheinung treten.
} 
zum Ausdruck gebracht werden, dominieren auch die demoskopische [öffentliche Meinung]“ (Schweiger und Weihermüller 2008, S. 555; vgl. Abschnitt 2). ${ }^{3}$

Anders als Umfragedaten eignet sich eine Untersuchung von Online-Kommentaren für die Untersuchung der Begründungspraxen, die in Diskussionen über die Besteuerung von Erbschaften eine Rolle spielen. Bei der Auswertung der Leserkommentare hat uns dementsprechend vor allem interessiert, mit welchen argumentativen Repertoires die Gegner und Befürworter der Erbschaftssteuer ihre Positionen jeweils zu stützen versuchen.

Forschungen zur Rechtfertigung von Vermögensübertragungen liegen bisher nicht vor. Eine solche „Vermessung“ des öffentlichen Diskurses würde jedoch erlauben, die dominierenden moralischen, gesellschaftspolitischen und ökonomischen Rechtfertigungen im Zusammenhang mit der Erbschaftsbesteuerung besser zu verstehen. Vermögensvererbung ist eine zentrale Institution der intergenerationalen Perpetuierung sozialer Ungleichheit und insofern von großem Interesse für die soziologische Forschung - die sich des Themas während der letzten 20 Jahre zwar vermehrt angenommen, dessen Bedeutung aber keineswegs erschöpft hat. Die Erbschaftssteuer ist dabei ein seit 150 Jahren diskutiertes Vehikel zur Reduzierung von Vermögensungleichheit. Die argumentativen Muster zu verstehen, die in der Bevölkerung in Hinblick auf die Erbschaftssteuer vorherrschen, ist nicht zuletzt auch für mögliche Reformvorschläge relevant, da erkennbar wird, welche Aspekte der Steuer in der Gesellschaft als besonders problematisch oder als besonders wichtig wahrgenommen werden. Die Auswertung der Online-Kommentare soll also zum Verständnis beitragen, wie deutsche und österreichische Bürger die Institution der Erbschaftsbesteuerung wahrnehmen und bewerten und so zu einem stärker reflektierten Umgang mit dem Thema beitragen.

Im Nachfolgenden legen wir zunächst unser Vorgehen bei der Auswertung der Leserkommentare dar, bevor wir in Abschnitt 3 den Diskurs von Gegnern und Befürwortern analysieren. In Abschnitt 4 zeigen wir anhand einer Faktorenanalyse die Zusammenhänge der unterschiedlichen Argumentationen auf. Die Ergebnisse diskutieren wir im Schlussteil.

\section{Vorgehen der Auswertung}

Der Diskurs um Erbschaftssteuern wurde anhand von Online-Kommentaren in öffentlichen Diskussionsforen der Webauftritte Standard.at und Spiegel Online ausgewertet. Aufhänger der Diskussion waren zwei Interviews zum Thema Erbschaftssteuern. Das erste wurde in der österreichischen Zeitung Der Standard mit dem Erstautoren dieses Berichts, Jens Beckert, geführt und am 18. Dezember 2014 online veröffentlicht (Bruckner 2014). Das zweite Interview wurde mit der Autorin Julia

\footnotetext{
3 Ein mögliches alternatives Vorgehen wäre etwa die Durchführung von Fokusgruppen gewesen, in denen ausgewählte Personen ihre Argumentationsmuster zur Erbschaftssteuer offengelegt hätten. Für ein solches Vorgehen bei der Untersuchung von Einstellungen zu Steuern (darunter der Erbschaftssteuer) in Großbritannien, siehe Prabhakar (2012). Stark et al. (2016) erheben und analysieren zudem freie Assoziationen zu Erbschaft und Vermögen aus der Perspektive der ökonomischen Verhaltensforschung.
} 
Friedrichs anlässlich des Erscheinens ihres Buches Wir Erben (Friedrichs 2015) geführt und am 14. März 2015 auf Spiegel Online veröffentlicht (Hucht 2015). Beide Interviews riefen in kurzer Zeit viele Reaktionen hervor. Auf das Interview im Standard gab es über 1600 Leserreaktionen, auf Spiegel Online wurden in zwei Tagen über 350 Kommentare abgegeben. Das so verfügbare Datenmaterial bietet sich zur Erforschung des Diskurses über die Erbschaftssteuer an.

Dabei sind jedoch zunächst einige Einschränkungen zu berücksichtigen. So ist es für die Inhaltsanalyse von Online-Kommentaren relevant zu berücksichtigen, wie die Nutzung des Internets, politische Online-Partizipation und Online-Diskussionen zu Selektivität führen. ${ }^{4}$ Wichtig ist zudem, dass in Online-Diskussionen üblicherweise ein Großteil der Kommentare von wenigen Nutzern abgegeben wird. Auch in unserer Auswertung wurden zum Beispiel in der Diskussion auf Standard.at etwa $50 \%$ der Kommentare von $10 \%$ der Kommentatoren abgegeben. ${ }^{5}$

Untersuchungen zu Online-Diskursen, wie zum Beispiel die Untersuchung von Schweiger und Weihermüller (2008), lassen indes vermuten, dass Online-Diskurse sich nur wenig von öffentlichen Diskursen offline unterscheiden. Besonders interessant für unser Vorhaben ist auch, dass Haas et al. (2010, S. 261) zeigen konnten, dass sich die Meinungsverteilung von Kommentatoren auf Spiegel Online zur Erbschaftssteuer nicht signifikant von der Verteilung in der Gesamtbevölkerung unterschied. ${ }^{6}$ Aufgrund dieser Ergebnisse nehmen wir an, dass sich auch die von uns untersuchten Online-Diskurse zur Erbschaftssteuer - trotz der ungleichen Aktivität der Kommentatoren - nicht grundsätzlich von aggregierter öffentlicher Meinung und öffentlichem Diskurs unterscheiden.

Zur Erfassung des Diskurses knüpfen wir methodisch an die quantitative Inhaltsanalyse von Parlamentsdebatten zum Thema Erbschaft bei Beckert (2004) an. Ziel der Inhaltsanalyse ist es, die in den Kommentaren verwendeten Argumente zu erfassen. Codiereinheiten sind die in den Kommentaren hermeneutisch erkennbaren Argumente zur Begründung einer Position. Zudem wurde die grundsätzliche Einstellung des Kommentierenden zur Erbschaftssteuer als befürwortend, ablehnend oder neutral erfasst. Die Argumente werden einzeln erfasst, wobei das Kategorien-

\footnotetext{
4 So zitieren zum Beispiel Harris et al. (2014, S. 1312-1313) verschiedene Studien zur Repräsentativität von Online-Diskussionen auf News-Webseiten, die für die USA nahelegen, dass die Nutzer von OnlineNews-Webseiten sich von der Gesamtbevölkerung vor allem durch das Alter, aber auch durch Herkunft, Geschlecht, Einkommen und Bildungsgrad unterscheiden. Zudem zeigt etwa Strandberg (2008) anhand repräsentativer Daten für Finnland, dass Online-Kommentatoren zur finnischen Wahl tendenziell auch außerhalb des Internets politisch aktiv sind und im Vergleich zu allgemeinen Internetnutzern in Finnland politisch sehr interessiert sind. Menschen, die online partizipieren, ,tend to be more knowledgeable about political issues, more involved in civic affairs offline, and as a result contribute knowledgeable viewpoints online“ (Harris et al. 2014, S. 1313). Ähnliche Ergebnisse zur politischen Online-Partizipation im Allgemeinen wurden zum Beispiel von Weber et al. (2003) oder Best und Krueger (2005) für die USA oder von Escher (2013) für Großbritannien aufgezeigt.

5 Eine solche Verteilung ist nicht ungewöhnlich und deckt sich zum Beispiel mit den Zahlen bei Blom et al. (2014, S. 1322; $47 \%$ der Kommentare durch $10 \%$ der Kommentatoren) oder bei Fuchs (2006, S. 14; $51 \%$ der Kommentare auf $12 \%$ der Kommentatoren).

6 In dieser Studie ging es dabei nicht um die Erforschung der Strukturen des Diskurses zur Erbschaftssteuer. Vielmehr ging es um die Methodenfrage der Vergleichbarkeit der Kommunikation online und offline, wofür die Erbschaftssteuer als eines von sechzehn Themen herangezogen wurde.
} 
Tab. 1 Kategoriensystem zur Erfassung der Argumente für oder gegen die Erbschaftssteuer

\begin{tabular}{|c|c|c|c|}
\hline \multicolumn{4}{|c|}{ Gründe: } \\
\hline 1 & Gleichheitsprinzip & 9 & $\begin{array}{l}\text { Zwischen Einkommensarten umver- } \\
\text { teilen }\end{array}$ \\
\hline 2 & Gerechtigkeitsprinzip & 10 & Bezug auf Wohneigentum \\
\hline 3 & Leistungsprinzip & 11 & Rahmenbedingungen \\
\hline 4 & $\begin{array}{l}\text { Eigentumsprinzip/Bewahrung von } \\
\text { Vermögen in der Familie }\end{array}$ & 12 & Wirtschaftsbezug \\
\hline 5 & Neid & 13 & Doppelte Besteuerung \\
\hline 6 & $\begin{array}{l}\text { Unzufriedenheit mit Staat, Politik } \\
\text { und/oder Verwaltung }\end{array}$ & 14 & Staatshaushalt \\
\hline 7 & $\begin{array}{l}\text { Bezug auf Demokratie/öffentliche } \\
\text { Meinung }\end{array}$ & 15 & Bezug auf Praxis im Ausland \\
\hline 8 & Soziale Ungleichheit & 16 & Kein Argument/anderes Thema \\
\hline
\end{tabular}

system im Codierprozess induktiv entworfen und erweitert wird. Als Rahmen der Inhaltsanalyse wurde das ursprünglich bei Beckert (2004, S. 339-341) verwendete Kategoriensystem grundlegend zusammengefasst und im Prozess der Kategorienbildung in mehreren Schritten an die vorliegenden Daten angepasst. Ergebnis dieses ersten Arbeitsschritts sind 15 Kategorien zur Erfassung der Argumente, die in Abschnitt 3 an Beispielen beschrieben werden (vgl. Tabelle 1).

Ein Argument besteht aus Wörtern oder Sätzen, die in der Gesamtheit ihres Zusammenhangs einen Sinn ergeben. Argumente, die in einem Satz mehrfach vorkamen, wurden genau einmal pro Satz codiert. Auch mehrere unterschiedliche Argumente wurden je einmal pro Satz codiert. Zudem wurden Argumente immer in ihrem gesamten Sinnzusammenhang betrachtet, das heißt, teilweise wurden mehrere Sätze oder ein ganzer Kommentar als einzelnes Argument vercodet. Die Einteilung der zu codierenden Einheiten oblag den Codierern. Hatte ein Benutzer mehrere Kommentare abgegeben, wurde jeder Kommentar unabhängig von den anderen Kommentaren ausgewertet. Direkte Zitate oder Bezüge auf vorherige Kommentare wurden nur codiert, wenn sie Teil eines oder mehrerer Argumente im zitierenden Kommentar waren. Kommentare, die keine auswertbaren Argumente enthielten oder sich offensichtlich auf andere Themen als die Erbschaftssteuer bezogen (zum Beispiel auf die Einkommenssteuer), wurden mit der Kategorie Kein Argument/anderes Thema codiert und nicht in die Auswertung einbezogen.

Durchgeführt wurde die Inhaltsanalyse von zwei geschulten Mitarbeitern des Max-Planck-Instituts für Gesellschaftsforschung. Für die Codierung der Positionen wurde nach der Schulung ein Inter-Coder-Reliabilitätstest durchgeführt, der akzeptable Übereinstimmung für die Positionen ergab $(\mathrm{PA}=.76) .^{7}$ Für die weitere Gewährleistung der Reliabilität wurden sechs weitere Tests durchgeführt und signifikant unterschiedliche Kategorien erneut gemeinsam überarbeitet und diskutiert. Zudem wurden nach der ersten vollständigen Auswertung signifikant unterschiedlich vergebene Kategorien im Konsens erneut codiert. Insgesamt wurden so 914

\footnotetext{
${ }^{7}$ Die Inter-Coder-Reliabilität wurde nach Holsti berechnet.
} 
und damit etwa $25 \%$ der ausgewerteten Argumente erneut in Zusammenarbeit der beiden Codierer codiert.

Datenbasis für die Inhaltsanalyse bilden 1654 Online-Kommentare, die wir von der Redaktion des Standard.at erhalten haben. Dazu kommen 363 auswertbare Kommentare aus dem Diskussionsforum von Spiegel Online, die wir eigenständig erfasst haben. Alles in allem wurden 2017 Kommentare ausgewertet. Von diesen enthielten 1209 Kommentare $(60 \%)$ erkennbare Argumente und bezogen sich auf die Erbschaftssteuer. $40 \%$ der Kommentare konnten nicht ausgewertet werden, da sie sich entweder nicht auf die Erbschaftssteuer bezogen, kein Argument oder nur unqualifizierte, nicht codierbare Inhalte wie Beleidigungen enthielten. Diese Zahl ist vergleichbar mit den Ergebnissen anderer Online-Inhaltsanalysen, etwa der eines österreichischen politischen Diskussionsforums bei Fuchs (2006), in der 68,8 \% der Kommentare Argumente für die dargelegte Meinung enthielten. Insgesamt wurden aus den 1209 auswertbaren Kommentaren 3573 Argumente erfasst.

\section{Beschreibung des Diskurses}

Die abgegebenen Kommentare können zunächst nach ihrer allgemeinen Haltung zur Erbschaftssteuer eingeteilt werden. Die Kommentare lassen gegenüber der Erbschaftssteuer entweder Ablehnung, Zustimmung oder Neutralität erkennen. Die Auswertung ergibt, dass sich insgesamt $52 \%$ der Kommentare gegen die Erbschaftssteuer aussprechen und $38 \%$ dafür. Rund $10 \%$ lassen keine eindeutige Meinung erkennen (vgl. Tabelle 2).

Dabei sind einige Unterschiede zwischen den beiden Diskussionen zu erkennen, die durch landesspezifische Differenzen erklärt werden können. In Deutschland finden etwa $70 \%$ der Bevölkerung die Besteuerung von Erbschaften ,generell unfair“ (Schmidt 2015) und $65 \%$ sprechen sich gegen eine Erhöhung aus (Infratest 2013, S. 4). Demgegenüber liegt der Anteil ablehnender Kommentare für das Portal Spiegel Online mit knapp $60 \%$ etwas unter diesen Werten, der Anteil ablehnender

Tab. 2 Positionen zur Erbschaftssteuer

\begin{tabular}{lllllllll}
\hline Interview & \multicolumn{2}{l}{ Ablehnung } & Neutral & \multicolumn{4}{l}{ Zustimmung } & Total \\
& Abs. & Prozent & Abs. & Prozent & Abs. & Prozent & Abs. & Prozent \\
Spiegel & 185 & 59,5 & 30 & 9,6 & 96 & 30,9 & 311 & 100 \\
Standard & 447 & 49,8 & 87 & 9,7 & 364 & 40,5 & 898 & 100 \\
Total & 632 & 52,3 & 117 & 9,7 & 460 & 38 & 1209 & 100 \\
\hline
\end{tabular}

Tab. 3 Anzahl und Anteil der gebrachten Argumente nach Positionen

\begin{tabular}{lll}
\hline & Argumente & \\
Position & Anzahl & Anteil \\
Ablehnung & 1998 & 55,9 Prozent \\
Neutral & 275 & 7,7 Prozent \\
Befürworter & 1300 & 36,4 Prozent \\
Total & 3573 & 100,0 Prozent \\
\hline
\end{tabular}


Kommentare mit $31 \%$ etwas darüber. Für die österreichische Seite Standard.at entspricht die Verteilung der Kommentare auf einen Prozentpunkt genau den Werten der für Österreich repräsentativen Umfrage des Market Instituts (contra 49 \%, pro 39 $\%$, Market Institut 2014). Alles in allem stehen die österreichischen Kommentatoren der Erbschaftssteuer also etwas weniger kritisch gegenüber als die deutschen.

Es fällt jedoch auf, dass auf Standard.at mit 45,7 \% der Kommentare wesentlich öfter kein Argument erfasst werden konnte oder sich auf ein anderes Thema bezogen wurde als auf Spiegel Online mit 14,6 \% der Kommentare. Möglicherweise deutet dies darauf hin, dass die Diskussion emotionaler geführt wurde und der Anteil unsachlicher Kommentare, insbesondere die unsachliche Bezugnahme auf andere Kommentare, größer ist. Eine weitere Erklärung könnte in den Unterschieden der technischen Funktionsweise der beiden Foren liegen: Während im Forum auf Standard.at direktes Antworten auf andere Kommentare möglich war und sich so häufiger ,Zwiegespräche“ und damit einhergehend Themenwechsel ergaben, war dies bei Spiegel Online nicht möglich. Ähnlich der Ausrichtung der Kommentare verteilen sich auch die in den Diskussionen gebrauchten Argumente (vgl. Tabelle 3).

Nicht nur sind zustimmende und ablehnende Argumente ungleich verteilt, sondern auch die verschiedenen Begründungen, auf die dabei jeweils zurückgegriffen wird. Befürworter und Gegner benutzen die verschiedenen, im Diskurs auffindbaren Rechtfertigungen unterschiedlich häufig und auf unterschiedliche Weise. Im Folgenden wird deren Verteilung deshalb für Befürworter und Gegner der Erbschaftssteuer getrennt beschrieben. Die neutrale Position wird aufgrund ihres geringen Auftretens und ihres ambivalenten Charakters vernachlässigt.

\subsection{Argumentation der Gegner}

Insgesamt wurden von den Gegnern der Erbschaftssteuer 1998 auswertbare Argumente vorgebracht. Die meisten davon beziehen sich im weitesten Sinne auf Rahmenbedingungen, die gegen Erbschaftssteuern sprechen (19\%), auf das Recht auf Eigentum (16\%) sowie auf das Leistungsprinzip (13\%). Darüber hinaus sind Argumente wichtig, die eine allgemeine Unzufriedenheit mit dem Staat, Politikern oder

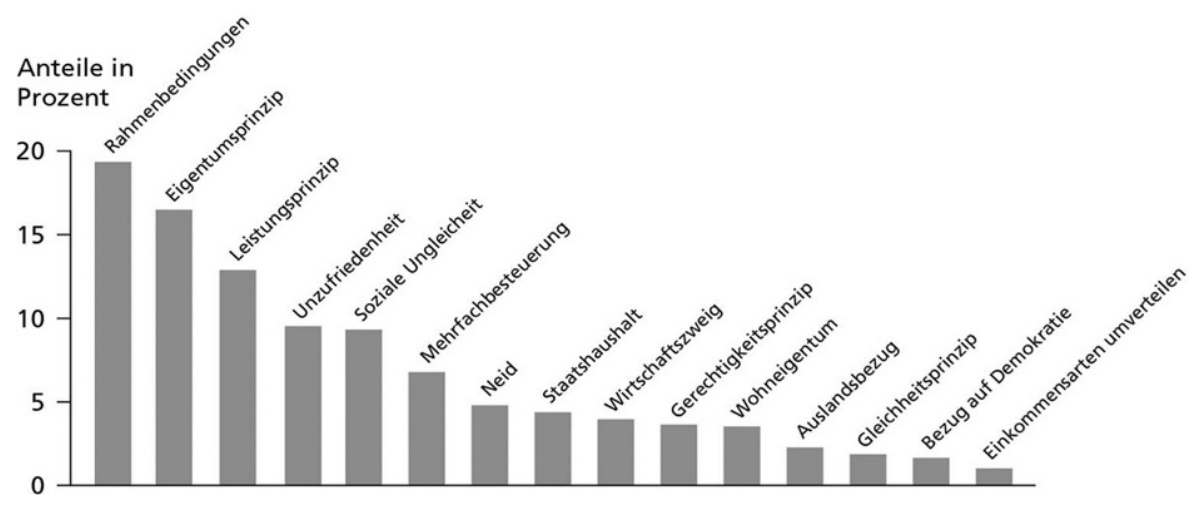

Abb. 1 Argumente der Gegner 
der Verwaltung zum Ausdruck bringen (9\%), die sich auf die Sozialstruktur und soziale Ungleichheit beziehen (9\%) oder die in der Erbschaftssteuer eine Mehrfachbesteuerung sehen (7\%). Die übrigen Varianten machen jeweils weniger als $5 \%$ sämtlicher ausgewerteten Argumente der Gegner aus (vgl. Abbildung 1). Die Argumente in den verschiedenen Kategorien werden im Folgenden an typischen Beispielen erläutert.

\subsubsection{Rahmenbedingungen}

Sehr häufig beziehen sich die Gegner der Erbschaftssteuer auf gegen die Erbschaftssteuer sprechende Rahmenbedingungen (19\%). Dabei spielen vor allem die Wirksamkeit, die Umsetzbarkeit und die Akzeptanz von Erbschaftssteuern in der Bevölkerung eine Rolle. Bezüglich der Wirksamkeit wird häufig angezweifelt, dass die Erbschaftssteuer Vermögensungleichheit entgegenwirken kann, da Menschen mit großen Vermögen einen Weg finden könnten, sich der Steuer zu entziehen:

Was gegen eine Erbschaftssteuer spricht ist das fluchtbereite Kapital, sprich: Diejenigen, die es treffen soll, die entziehen sich. (Kommentar 150, Spiegel Online $)^{8}$

Außerdem ist es schwierig zwischen Wertgegenständen und Gebrauchsgütern zu unterscheiden. Die Besteuerung von Bildern oder Schmuck hängt davon $\mathrm{ab}$ welcher Kategorie sie zugeordnet werden. Der Wert von Wertgegenständen lässt sich nicht so einfach schätzen. Das sollte man wirklich nicht jedes Jahr tun. (Kommentar 1099, Standard.at)

Neben Fragen der Umsetzbarkeit wird außerdem die Stichhaltigkeit weiterer Annahmen diskutiert, die bei Begründungen der Erbschaftssteuer eine Rolle spielen. Im Interviewtext auf Spiegel Online wird etwa genannt, dass das Erben eine emotionale Belastung darstelle, etwa aufgrund des Gefühls, das Geld nicht verdient zu haben (Hucht 2015). Dies wird von den Gegnern jedoch nicht als Argument für die Erbschaftssteuer akzeptiert:

„Viele Erben fühlen sich schuldig“Dann sollen sie halt auf das Erbe verzichten, dann sind die Schuldgefühle gleich weg. (Kommentar 43, Spiegel Online)

Charakteristisch für die genannten Argumente ist generell, dass hiermit die Erbschaftssteuer nicht unmittelbar normativ bewertet, sondern vielmehr die Beeinträchtigung von Effektivität und Effizienz einer solchen Steuer durch diverse Rahmenbedingungen diskutiert wird.

\footnotetext{
${ }^{8}$ Die Kommentare werden hier jeweils ohne irgendwelche Korrekturen wiedergegeben. Die Rechtschreibfehler und häufigen Betonungen etwa durch Großschreibung ganzer Wörter geben einen Eindruck von der Flüchtigkeit, mit der die Autoren teilweise kommentiert haben, aber auch von ihrer emotionalen Involviertheit. Die Kommentare auf Spiegel Online können anhand der dargestellten Nummerierung so online nachgelesen werden. Die Kommentare auf Standard.at konnten von uns jedoch nur nach ihrem zeitlichen Auftreten sortiert und nummeriert werden, sodass die Nummerierung hier nicht der im öffentlichen Forum entspricht.
} 


\subsubsection{Recht auf Eigentum}

Eines der wichtigsten inhaltlichen Argumente der Gegner der Erbschaftssteuer ist der Verweis auf das individuelle Eigentumsrecht (16\%). Das eigene Vermögen wird als Privatsache gesehen, in die sich der Staat nicht einzumischen hat. Oft fallen auch Vergleiche mit Enteignung oder Kommunismus.

Es ist eine Frechheit, es allen Ernstes für fair zu finden dass der Staat nach meinem Tod mein Vermögen krallt. (Kommentar 134, Spiegel Online)

Diese - häufig in emotionaler Sprache vorgebrachten - Argumente verweisen auf ein Gefühl der Bedrohung der eigenen Sparleistungen und Arbeitsanstrengungen durch die Erbschaftssteuer und werden häufig auch auf die eigene Familie bezogen. Hier äußert sich ein für Deutschland typisches, bis in das 19. Jahrhundert zurückzuverfolgendes Argumentationsmuster gegen die Erbschaftsbesteuerung (vgl. Beckert 2004). Die Kommentierenden geben an, sich das Vermögen aufgebaut zu haben, um es an ihre Angehörigen weiterzugeben. Eine Abführung von Steuern an den Staat betrachten sie dagegen als Benachteiligung ihrer Familien.

Wenn mir meine Vorfahren ihre Früchte geben wollen habe ich natürlich das

Recht darauf, ist ja ihr Geld. (Kommentar 1060, Standard.at)

Das Recht auf „Familieneigentum“ wird von den Gegnern der Erbschaftssteuer stark in den Vordergrund gerückt. Der Erblasser sollte alleine beziehungsweise im Sinne der Familie über den Transfer des Eigentums entscheiden können. Der Staat habe kein Recht, sich in den Vermögensübergang einzumischen.

\subsubsection{Leistungsprinzip}

Ein weiteres wichtiges Argument der Gegner der Erbschaftssteuer bezieht sich auf die Frage nach der Legitimation der leistungslosen Erlangung von Vermögen durch Erbschaft (13\%). Anzuerkennen seien die Leistungsfähigkeit und Leistungsbereitschaft von Erblassern, die zur Verfügung über das Vermögen berechtigten. Der Bezug auf das Leistungsprinzip findet etwa auch darin Ausdruck, Personen, denen geringere Leistungsbereitschaft zugeschrieben wird, vorzuwerfen, sich das Vermögen der Leistungserbringer leistungslos über die Erbschaftssteuer aneignen zu wollen:

Sorry - alle die ständig nach dem Geld anderer schreien, sollten zuerst einmal einen Beruf erlernen, mit dem sich Geld verdienen lässt und dann 10-20 Jahre 70-80 Stunden die Woche arbeiten. (Kommentar 9, Spiegel Online)

Häufig wird dabei auch der Verzicht auf Konsum genannt. Es wird ein Bild vom sparsamen Arbeiter gezeichnet, der sich durch Verzicht ein Vermögen aufgebaut hat, etwa um es an die eigenen Kinder weiterzugeben. Teilweise bezieht sich dieses Bild negativ auf eine behauptete andere soziale Gruppe, die durch übermäßigen Konsum nichts angespart habe, also die Leistung des Verzichts nicht erbracht hat. Die Befürworter der Erbschaftssteuer werden so moralisch desavouiert: 
Dafür haben Sie auch gearbeitet und vielleicht auf etwas Urlaub oder teureren Urlaub oder vielleicht auf einen neuen Fenrseher alle zwei Jahre verzichtet. Andere, die lieber Urlaub machen, ständig shoppen gehen usw. sparen sich dann die Erbschaftssteuer auf Vermögen und fordern, dass der Staat noch mehr Geld abkassiert. (Kommentar 146, Spiegel Online)

Ein dritter, eher makroökonomischer Aspekt ist die genannte Befürchtung, dass durch die Erbschaftssteuer das Leistungsprinzip verletzt wird:

Warum soll in dem land ueberhaupt noch wer arbeiten und was Leisten wenn einem am ende sowieso alles weggenommen wird. (Kommentar 792, Standard.at)

Die Gegner der Erbschaftssteuer beziehen sich demnach insbesondere auf drei verschiedene Weisen auf das Leistungsprinzip: auf die von den Erblassern erbrachten Leistungen, auf mit dem Vermögensaufbau verbundenen Einschränkungen des Konsums der Erblasser sowie auf die Gefahr der Demotivierung gesellschaftlicher Leistungserbringer durch die Besteuerung von Erbschaften.

\subsubsection{Weitere Argumente}

Eine nennenswerte Rolle bei der Begründung der Erbschaftssteuerablehnung spielt auch die Äußerung allgemeiner Unzufriedenheit mit dem Staat, Politikern oder der Verwaltung (9\%). Hier geht es zumeist um ein eher diffuses, teilweise jedoch auch direkt auf bestimmte Personen oder Parteien gemünztes Missfallen gegenüber staatlichen Akteuren, ,der Politik“ oder dem politischen Establishment:

Mir ist schon klar, dass ich nicht die Steuer bezahle, das ist für mich aber irrelevant, denn wenn ein Geld oder Vermögen im Steuersumpf verschwindet, dann trachte ich danach, kein Vermögen zu haben, das geerbt werden kann. (Kommentar 726, Standard.at)

Außerdem sind für die Gegner der Erbschaftssteuer deren erwartete Auswirkungen auf die Sozialstruktur und die Entwicklung sozialer Ungleichheit ein relevantes Thema (9 \%). Dabei wird gerade das Ziel der Umverteilung durch die Erbschaftssteuer kritisiert:

Das übliche Umverteilungsgerede jener die meinen vom Leben benachteiligt zu sein und glauben die Probleme würden sich lösen indem man den sog. „Reichen“ noch mehr wegnimmt. (Kommentar 201, Spiegel Online)

Darüber hinaus wird die Sorge geäußert, dass durch die Einführung der Erbschaftssteuer vor allem die Mittelschicht betroffen sein und am sozialen Aufstieg gehindert werden wird:

Und was die Umverteilung betrifft, wohl wahr, durch die immer höheren Belastungen der Mittelschicht, bzw. besser gesagt des Mittelbaus, wird es zu einer noch größeren Kluft kommen. (Kommentar 318, Standard.at) 
In dieser Argumentation zeigt sich sichtbar Unwissen über den Tatbestand, dass die Erbschaftssteuer aufgrund von hohen Freibeträgen eine Steuer ist, die überwiegend die Oberschicht betrifft.

Ein letztes Argument, das erwähnt werden soll, ist das Argument der Mehrfachbesteuerung (7\%). Dabei wird argumentiert, dass das Vermögen bereits vorher etwa mit der Einkommenssteuer und weiteren Steuern belastet wurde und eine Veranlagung bei der Vermögensübertragung somit die mehrfache Besteuerung desselben Vermögens bedeutet:

Mein Eigentum, dass schon vorher x-mal besteuert worden ist, wohlgemerkt! Das erste Mal beim Verdienen (Einkommensteuer), das zweite mal beim Umwandeln von Geld in Waren (Umsatzsteuer). Falls die Ware Grundeigentum ist, wird sogar jährlich besteuert (Grundsteuer). Falls ich es anlege, wird der Ertrag ebenfalls besteuert (Kapitalertragsteuer). Falls ich es einfach nur unters Bett packe, wird ebenfalls besteuert (Inflation). Seid ihr einfach nur zu feige, um gleich die Verstaatlichung allen Eigentums zu fordern? (Kommentar 300, Spiegel Online)

\subsection{Argumentation der Befürworter}

Die Befürworter der Erbschaftssteuer haben insgesamt 1300 Argumente und damit 36,4 \% aller Argumente zu den Diskussionen beigetragen. Genau wie bei den Gegnern sind die Bezüge auf Rahmenbedingungen die am häufigsten vorzufindende Argumentationsweise ( $21 \%$ ). Mit $15 \%$ bezieht sich ein weiterer bedeutender Anteil der Argumente jedoch, anders als bei den Gegnern, auf den Themenkomplex der sozialen Ungleichheit und Sozialstruktur. Das Leistungsprinzip ist auch in dieser Gruppe eines der wichtigsten Themen (15\%), wird jedoch anders interpretiert. Weiterhin wichtig sind Einschätzungen zur Mehrfachbesteuerung (10\%), die Forderung, zwischen den Einkommensarten umzuverteilen (7\%), sowie Argumente, die sich auf das Eigentumsprinzip beziehen (5\%; vgl. Abbildung 2). Wieder sollen im Folgenden die wichtigsten Argumenttypen anhand von Beispielen erläutert werden.

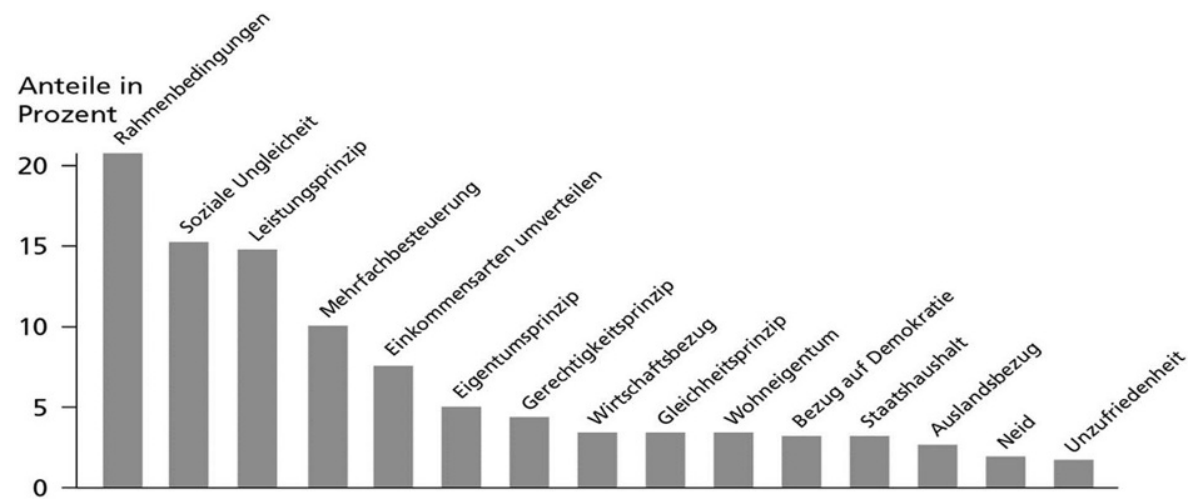

Abb. 2 Argumente der Befürworter 


\subsection{Rahmenbedingungen}

Die Befürworter der Erbschaftssteuer beziehen sich im Vergleich zu den Gegnern eher auf Umstände, die die Erbschaftssteuer stützen, wie etwa positive Einschätzungen zur Umsetzbarkeit sowie zur Wirksamkeit der Steuer und ihrer Akzeptanz. Prominent wird dabei auf Freibeträge eingegangen und darauf verwiesen, dass nur hohe Vermögen belastet würden - und somit nur die wenigsten von der Steuer betroffen wären:

Wenn die wirklich Reichen damit belastet werden ist das okay. Aber mit einem Reihenhaus in München hat man auch schon einen Wert von 600.000 Euro. Das ist aber kein Reichtum hier. Also hohe Freibeträge um die 1 Million Euro, die ab und zu angepaßt werden wäre richtig. Darüber könnte man schon mehr besteuern. (Kommentar 3, Spiegel Online) ${ }^{9}$

Darüber hinaus werden vor allem Argumente der Gegner aufgegriffen und gegensätzlich gewertet. Etwa das Argument der schwierigen Umsetzbarkeit:

Nur weil eine Maßnahme vielleicht schwer umzusetzen ist, heisst das nicht, dass sie deswegen schlecht ist. (Kommentar 131, Standard.at)

Oder aber gegen das Argument der Kapitalflucht ins Ausland durch die Erbschaftssteuer:

Man muss sich das nur trauen, die wenigsten werden abwandern, weil Österreich einiges zu bieten hat. (Kommentar 690, Standard.at)

Auch wird die Erbschaftssteuer mit anderen Steuern wie der Einkommenssteuer in Verbindung gebracht und auf die Notwendigkeit der Staatsfinanzierung verwiesen, zu der die Besteuerung von Erbschaften ihren Teil beiträgt:

Und das ganze ist dem Grundprinzip untergeordnet, dass ein ZUGEWINN versteuert werden muss Sei es Erbe, sei es Gehalt, sei es Schenkung (Kommentar 283, Spiegel Online).

Die Befürworter der Erbschaftssteuer weisen damit Argumente zurück, die von den Gegnern vorgebracht werden. D.h. die Rahmenbedingungen der Steuer werden von Gegnern und Befürwortern unterschiedlich bewertet. Insgesamt wird so für die Umsetzbarkeit, die Wirksamkeit und die soziale Akzeptanz der Steuer argumentiert.

\subsubsection{Soziale Ungleichheit und Sozialstruktur}

Der von den Befürwortern der Steuer am zweithäufigsten angeführte Komplex von Gründen ist der Umgang mit sozialer Ungleichheit und die Auswirkungen einer

\footnotetext{
${ }^{9}$ In diesem Fall wurden - wie in anderen auch - einzelne Teile des Kommentars in unterschiedliche Kategorien vercodet. Neben dem Verweis auf die Rahmenbedingungen betrifft das bei diesem Zitat die Kategorien Soziale Ungleichheit und Wohneigentum, allerdings jeweils bezogen auf unterschiedliche Teile (es wurde nie derselbe Teil eines Satzes in mehr als eine Kategorie vercodet). Dennoch zitieren wir hier die gesamte Passage, damit der Sinnzusammenhang deutlich wird.
} 
Erbschaftssteuer auf die Sozialstruktur (15\%). Der bedeutendste Punkt ist hier die Forderung nach Umverteilung und insbesondere nach dem Ausgleich allzu ausgeprägter Vermögensungleichheit. Die Erbschaftssteuer soll makrosozial zur Korrektur sozialer Ungleichheit beitragen:

Die Tatsache, dass Großvermögen seit Jahrzehnten bei uns steuerlich einzigartig privilegiert sind, hat zu großen gesellschaftlichen Verzerrungen und sozialen Schieflagen geführt (Kommentar 350, Spiegel Online).

Mit gleicher Zielrichtung, doch umgekehrten Vorzeichen wird eine bestehende Umverteilung ,,von unten nach oben“ beklagt:

Bei der beständigen Umverteilung von Arm nach Reich ist das Erben fast einer der gewichtigsten Faktoren. (Kommentar 96, Spiegel Online)

Bezug wird auch auf Armut in den ökonomisch benachteiligten Bevölkerungsschichten genommen:

Allerdings sollte niemand gezwungen sein, die Last seiner Geburt mitschleppen zu müssen und deshalb gegenüber anderen (aus vermögenderen oder sozial höher gestellten Familien) Nachteile zu haben. (Kommentar 552, Standard.at)

Von den Kommentaren wird eine fortschreitende Vermögenskonzentration bei Wenigen auf Kosten der unteren und mittleren Einkommens- und Vermögensschichten kritisiert. Um diese Entwicklung auszugleichen, wird die Besteuerung insbesondere der größten Erbschaften gefordert.

\subsubsection{Leistungsprinzip}

Das Leistungsprinzip ist auch für die Befürworter der Erbschaftssteuer ein wichtiges Thema. Für die Befürworter steht jedoch relativ einhellig als wichtigster Punkt des Leistungsprinzips der Gedanke im Vordergrund, dass Erbschaften Einkommen darstellen, welche die Erben nicht durch eigene Leistung verdient haben:

Nichts geleistet, nichts riskiert, bekommt $500000 €$ geschenkt und soll für das, was darüber hinaus geht, nicht ein mal ein paar Prozent Steuern zahlen, während der Schulkollege aus armen Verhältnissen für das selbst Erarbeitete fast 50 Prozent Lohnsteuer blechen muss? (Kommentar 508, Standard.at)

Dieser Gedanke wird in vielfältiger Form geäußert. Gefordert wird die Durchsetzung des Leistungsprinzips durch die Einführung der Erbschaftssteuer. Andere mit Leistung verbundene Aspekte tauchen bei den Befürwortern hingegen kaum auf.

Den Befürwortern der Erbschaftssteuer geht es nicht um die Befürchtung, dass die Einführung der Erbschaftssteuer zu geringerer Leistungsbereitschaft führt. Auch wird im Gegensatz zu den Gegnern der Steuer die Leistungslosigkeit beim Erbenden als wichtiger erachtet als der durch Leistung erworbene Anspruch des Erblassers, auch nach dem Tod über das Vermögen bestimmen zu können. Stattdessen wird kritisiert, dass Menschen durch Erbschaften Vermögen bilden können, ohne entsprechende Leistungen erbracht zu haben. 


\subsubsection{Weitere Argumente}

Drei weitere Argumentkategorien vereinen jeweils mehr als $5 \%$ der Nennungen auf sich. Beim Thema Mehrfachbesteuerung (10\%) wird von den Befürwortern der Erbschaftssteuer etwa argumentiert, dass diese keine solche darstellt, da die Steuer beim Erben und nicht beim Erblasser erhoben wird:

Erbschaftsvermögen wurde nicht zig mal besteuert - Der Erbe hat dafür ja noch nie Steuer gezahlt. (Kommentar 911, Standard.at)

Eine weitere prominente Forderung bei den Befürwortern der Steuer ist die Umverteilung der Steuerlast zwischen den Einkommensarten (7\%). Entweder soll die Steuer auf Arbeit gesenkt oder eine Erbschaftssteuer in ähnlicher Höhe eingeführt werden:

Arbeit und Erben gleich hoch besteuern wäre absolut richtig was ich nicht verstehen kann: viele glauben, sie hätten Anspruch auf die Früchte der Leistungen ihrer Vorfahren - gleichzeitig ist es aber ganz selbstverständlich keinen Anspruch auf den vollen Arbeitslohn zu haben, für den man hart gearbeitet hat (Kommentar 1145, Standard.at).

Insbesondere von den Kommentierenden auf Standard.at wird dieses Argument gebracht, wohl auch weil es im Interview explizit als Argument angeführt wird.

Auch die Befürworter der Erbschaftssteuer beziehen sich auf das Eigentumsprinzip und Eigentumsverständnis in der Gesellschaft (5\%). Hier wird jedoch eher in den Fokus gerückt, dass das Eigentumsverständnis gesellschaftlich geprägt ist und Eigentum auch verpflichtet:

Im Grundsatz geht es um die Frage, welchen Teil gibt der einzelne Staatsbürger, von seinem in seiner Verfügungsgewalt befindlichem Gut an die Allgemeinheit ab. (Kommentar 204, Standard.at)

Der Verweis auf die notwendige Finanzierung des Gemeinwesens wird als Rechtfertigung der Erbschaftssteuer angeführt, das Prinzip unbeschränkter individueller Eigentumsrechte dagegen in Zweifel gezogen.

\section{Argumentationsmuster im Diskurs}

Nachdem der Diskurs und die genannten Argumente beschrieben wurden, zeigen wir im nächsten Abschnitt typische Argumentationsmuster mithilfe einer explorativen Faktorenanalyse auf. Wir gehen dabei davon aus, dass es unabhängig von der Position zur Erbschaftssteuer auftretende Argumentzusammenhänge gibt. Diese werden sowohl von Gegnern als auch von den Befürwortern verwendet - zum Beispiel indem direkt auf einen Kommentar der anderen Position eingegangen wird. 


\subsection{Argumentationsmuster}

\subsubsection{Faktorenanalyse}

Die in der Inhaltsanalyse ermittelten Begründungen dienen als Datengrundlage für die Faktorenanalyse. Die Kommentare mit neutraler Position wurden auch hier ausgeschlossen, da wir die neutralen Kommentare aufgrund ihrer geringen Fallzahl für zwar besondere, aber vernachlässigbare Fälle halten. Für die 1092 einbezogenen Kommentare wurden die vorkommenden Argumente aufsummiert und die Summen je Kommentar verwendet. Das Kaiser-Meyer-Olkin-Kriterium misst die Güte der Ausgangsdaten zur Durchführung einer Faktorenanalyse und liegt mit 0,52 über dem geforderten Mindestwert (Kaiser 1974). Als Schätzverfahren verwenden wir die Hauptkomponentenanalyse. Zudem wurde eine rechtwinklige Varimax-Rotation mit Kaiser-Normalisierung durchgeführt, da wir nicht zwingend davon ausgehen, dass die Faktoren untereinander korrelieren (Backhaus et al. 2016, S. 413). Zur Ermittlung der Faktorenanzahl wurden in einem Scree-Test Faktoren mit Eigenwerten größer 1 berücksichtigt sowie die theoretische Plausibilität herangezogen.

Mit der Faktorenanalyse werden anhand der Daten zunächst Argumentationsmuster identifiziert, welche die Struktur der Gesamtheit der Kommentare abbilden. Dabei wird die Autorenschaft der Kommentare ignoriert und das gesamte Material als Abbild des Diskurses verstanden, die Kommentare also als unabhängige Diskursbeiträge behandelt. Die identifizierten Muster sind dann als Kombinationen von Argumenten zu verstehen, die sowohl von Befürwortern als auch von Gegnern der Steuer besonders häufig oder besonders selten zusammen genannt werden. Sie beschreiben Komplexe von Begründungen, die in unserer Auswertung der Online-Diskurse unterscheidbar identifiziert werden können. So werden beispielsweise die Argumente „Wohneigentum“ und „Wirtschaftsbezug“ relativ häufig mit Bezug auf ,Soziale Ungleichheit“ genannt, jedoch eher selten zusammen mit dem Argument der doppelten Besteuerung. Daraus lässt sich schlussfolgern, dass in diesem Argumentationsmuster vor allem makrosoziale Fragen diskutiert werden, dabei jedoch etwa die Diskussion um doppelte Besteuerung auffällig selten berührt wird und Fragen der Umsetzbarkeit vernachlässigt werden. Die Ergebnisse sollen zum Verständnis dazu beitragen, wie die einzelnen Begründungen für oder gegen die Erbschaftssteuer im öffentlichen Diskurs zusammenhängen.

\subsubsection{Ergebnisse}

Nach dem beschriebenen Verfahren ergibt sich die insgesamt plausibelste Lösung in Form von fünf Argumentationsmustern (Faktoren), die zusammen $41 \%$ der kumulierten Varianz erklären (vgl. Tabelle 4). Im Folgenden werden die ermittelten Muster kurz beschrieben und mit den Ergebnissen der Inhaltsanalyse in Verbindung gebracht.

Wertorientiertes Muster: Das erste Muster zeichnet sich dadurch aus, dass vor allem Werte wie Gleichheit, Leistung und das Eigentumsprinzip zur Begründung angeführt werden. Insbesondere das Leistungsprinzip nimmt hier einen wichtigen Stellenwert ein. Für die Dominanz von Wertorientierungen spricht auch, dass prag- 
Tab. 4 Argumentationsmuster in der Diskussion als Ergebnis der Faktorenanalyse

\begin{tabular}{llllll}
\hline Grund & $\begin{array}{l}\text { Wertorien- } \\
\text { tiert }\end{array}$ & Makrosozial & $\begin{array}{l}\text { Unzufriedenheit } \\
\text { und Misstrauen }\end{array}$ & Neid & $\begin{array}{l}\text { Eigentums- } \\
\text { bewahrend } \\
-0,31\end{array}$ \\
$\begin{array}{l}\text { Gleichheitsprinzip } \\
\text { Gerechtigkeitsprinzip }\end{array}$ & 0,39 & & & 0,54 & \\
$\begin{array}{l}\text { Leistungsprinzip } \\
\text { Eigentumsprinzip }\end{array}$ & $\mathbf{0 , 6 4}$ & 0,35 & & & 0,53 \\
$\begin{array}{l}\text { Neid } \\
\text { Unzufriedenheit }\end{array}$ & & $\mathbf{0 , 7 3}$ & $\mathbf{0 , 6 3}$ & \\
$\begin{array}{l}\text { Demokratie } \\
\text { Soz. Ungleichheit }\end{array}$ & 0,30 & & 0,55 & \\
$\begin{array}{l}\text { Einkommensarten } \\
\text { Wohneigentum }\end{array}$ & $\mathbf{0 , 6 6}$ & & & \\
$\begin{array}{l}\text { Rahmenbedingungen } \\
\text { Wirtschaftsbezug }\end{array}$ & $\mathbf{- 0 , 6 3}$ & 0,50 & & $\mathbf{- 0 , 6 5}$ \\
$\begin{array}{l}\text { Doppelte Besteue- } \\
\text { rung }\end{array}$ & & 0,53 & & & \\
$\begin{array}{l}\text { Staatshaushalt } \\
\text { Auslandsbezug }\end{array}$ & $-0,35$ & & & \\
\hline
\end{tabular}

Faktorladungen als Ergebnis der Faktorenanalyse durch Hauptkomponentenanalyse und nach orthogonaler Varimax-Rotation mit Kaiser-Normalisierung. Faktorladungen kleiner I.3। sind nicht aufgeführt. Faktorladungen größer gleich $1.3 \mathrm{I}$ und kleiner I.6I sind kursiv hervorgehoben. Faktorladungen größer gleich I.6I sind fett dargestellt.

matische Überlegungen wie die Umsetzbarkeit oder Wirksamkeit der Erbschaftssteuer in Form von Rahmenbedingungen in diesem Muster verstärkt ausgeblendet werden.

Makrosoziales Muster: Im zweiten ermittelten Muster spielen vor allem Argumente eine Rolle, die sich auf die Auswirkungen der Erbschaftssteuer auf makrosoziale Aspekte beziehen. Hier sind vor allem die beschriebenen Argumente zur sozialen Ungleichheit und Sozialstruktur zu nennen, aber auch die Auswirkungen der Erbschaftssteuer auf die Wirtschaft, zum Beispiel durch Auszahlungsansprüche von Erben oder mögliche Belastung von Familienunternehmen. Auch die Frage, ob eigengenutztes Wohneigentum unter die Erbschaftssteuer fallen würde und sollte, sowie Auswirkungen auf die Demokratie und öffentliche Meinung werden in diesem Zusammenhang diskutiert. Diskussionen, ob die Erbschaftssteuer eine Mehrfachbesteuerung darstellt, sind in diesem Muster besonders selten vorzufinden.

Unzufriedenheit und Misstrauen: Dieses Argumentationsmuster zeichnet sich durch den Ausdruck von Unzufriedenheit mit dem Staat, Politikern oder der Verwaltung aus. In diesem Zusammenhang werden auch intensiv finanzpolitische Aspekte diskutiert, die etwa die Auswirkungen der Erbschaftssteuer auf den Staatshaushalt, den richtigen Umgang mit Haushaltsmitteln oder die aktuelle Ausrichtung der Finanzpolitik betreffen.

Neid: Das vierte Muster wird sehr stark von der Diskussion um Neid geprägt - verbunden mit grundsätzlichen Erwägungen zur Gerechtigkeit der Erbschaftsbesteuerung oder zu deren Auswirkungen auf die Stabilität der Demokratie. Diskutiert 
wird etwa, ob die Erbschaftssteuer aus Neid gefordert wird, oder ob Neid eine irreführende Kategorie ist, an deren Stelle es um andere Zusammenhänge gehen sollte.

Eigentumsbewahrend: Das fünfte Muster ist geprägt durch die Hervorhebung des Eigentumsprinzips. Keine Rolle spielen dabei insbesondere Forderungen nach der Umverteilung zwischen den Einkommensarten Arbeit und Vermögen, was den Schluss zulässt, dass bestehende Eigentumsverhältnisse gerechtfertigt werden. Dementsprechend wird auch das Gleichheitsprinzip seltener angeführt. Die Praxis im Ausland wird mitdiskutiert, etwa in Hinsicht auf nicht vorhandene Erbschaftssteuern in anderen Staaten, z.T. wird auch auf nicht mehr existierende Staaten wie etwa die DDR oder die Sowjetunion verwiesen.

In der Faktorenanalyse konnten wir diese fünf Argumentationsmuster unterscheiden. Damit geht allerdings noch keine Differenzierung zwischen ablehnenden und zustimmenden Haltungen einher. Derartige strukturelle Argumentationsunterschiede zwischen Gegnern und Befürwortern der Erbschaftssteuer zeigt eine ausführlichere Auswertung mithilfe einer Clusteranalyse (vgl. auch Beckert und Arndt 2016).

Bei den Befürwortern sticht dabei kein einzelnes der zuvor genannten Argumentationsmuster besonders heraus. Sie zeichnen sich vielmehr dadurch aus, dass sie bestimmte Argumentationsmuster auffällig häufig nicht verwenden, nämlich die Muster „Eigentumsbewahrend“ sowie „Unzufriedenheit und Misstrauen“. Die Argumentationsmuster, die bei den Gegnern eine besonders wichtige Rolle spielen, sind bei den Befürwortern wiederum nur durchschnittlich vertreten.

Bei der Gruppe der Gegner lassen sich dagegen drei Gruppen unterscheiden: erstens die „unzufriedenen Gegner“, bei denen überwiegend die Unzufriedenheit und das Misstrauen gegen staatliche Institutionen im Vordergrund steht; zweitens „wertorientiert-bewahrende Gegner“, für die insbesondere das Argument, dass die Erbschaftssteuer ein illegitimer Eingriff in Familieneigentum ist, sowie der Verweis auf die Leistungen des Erblassers und das den Befürwortern der Steuer unterstellte Neidmotiv im Vordergrund stehen; sowie drittens eine Gruppe ,makrosozial argumentierender Gegner", die vor allem auf eine Benachteiligung der Mittelschicht und Beeinträchtigung sozialer Mobilität abheben sowie negative Auswirkungen auf Wohneigentum, die wirtschaftliche Entwicklung oder die Demokratie befürchten (siehe detailliert dazu Beckert und Arndt 2016).

\section{Diskussion und Schlussfolgerung}

Ziel der vorgenommenen Auswertung von zwei Online-Diskussionsforen zum Thema Erbschaftsbesteuerung war es, einen Eindruck der Strukturen des Diskurses zur Erbschaftsbesteuerung in Deutschland und Österreich zu gewinnen. Während die grundsätzliche Einstellung zu der Steuer aus Meinungsumfragen bekannt ist, wurden die für die Ablehnung oder Befürwortung angegebenen Gründe bisher nicht untersucht.

Die vorgenommene inhaltsanalytische Auswertung zeigt zunächst einmal, dass in beiden Ländern überwiegend Position gegen die Erbschaftssteuer bezogen wird. Dieses Resultat entspricht den bekannten Ergebnissen aus Meinungsumfragen. Bei den Begründungen der jeweiligen Positionen wird deutlich, dass beide Seiten häufig auf 
Rahmenbedingungen verweisen. Für die Gegner der Erbschaftssteuer geht es dabei um Umstände, die nach ihrem Dafürhalten die Erhebung der Steuer ineffektiv oder ineffizient erscheinen lassen, da mit Verlagerungsstrategien gerechnet werden müsse. Zudem werden unlösbare Bewertungsfragen ebenso wie die mutmaßlich fehlende gesellschaftliche Akzeptanz der Steuer angeführt. Die Befürworter auf der anderen Seite beziehen sich sogar in etwas höherem Maße auf Rahmenbedingungen, ziehen dabei aber naturgemäß andere Zusammenhänge heran. So sei die Erhebung der Steuer praktisch möglich, die Steuer sei ein wirksames Instrument der Steuervereinnahmung, sie würde akzeptiert (zumindest soweit nur die wirklich großen Vermögen besteuert würden) und die Notwendigkeit der Erzielung von Staatseinnahmen mache die Steuer sinnvoll.

Hinsichtlich der unmittelbar normativen Begründungen verweisen die Gegner der Erbschaftssteuer insbesondere auf das Recht der Vererbung von Eigentum als liberalem Grundrecht und auf die Leistungen des Erblassers, die durch die Besteuerung des Erbes geradezu bestraft würden. Außerdem rekurrieren die Gegner auf eine allgemeine Unzufriedenheit mit den staatlichen Institutionen und das technische Argument einer angeblichen Doppelbesteuerung, da der Erblasser das Vermögen ja bereits versteuert habe. Die Befürworter der Steuer verwenden hingegen völlig andere Rechtfertigungsmuster. So steht bei ihnen die Problematik sozialer Ungleichheit im Mittelpunkt, die durch die Erbschaftssteuer korrigiert werden könne. Auch sie beziehen sich auf das Leistungsprinzip, interpretieren dies jedoch ganz anders: Nicht die Leistungen des Erblassers, sondern die Leistungslosigkeit der Erben wird in den Vordergrund gerückt. Darüber hinaus wird das Argument der Mehrfachbesteuerung zurückgewiesen und beim Eigentumsprinzip die Sozialverpflichtung des Eigentums hervorgehoben.

Sowohl bei Gegnern wie bei Befürwortern fällt auf, dass einige Argumente kaum eine Rolle spielen, die in Stellungnahmen von Interessenverbänden und Experten regelmäßig angeführt werden. Das gilt vor allem für die Frage der Folgen der Erbschaftsbesteuerung für Unternehmen. Dies ist überraschend, weil zumindest im Interview im Standard dieses Thema ausführlich erörtert wurde. Die Ursache hierfür könnte in der sozialen Position der Kommentierenden liegen: Die Anliegen von Unternehmern berühren die Kommentatoren nicht und liegen außerhalb ihres eigenen unmittelbaren Interessenbereichs. Ein anderer Grund könnte sein, dass zumindest in Österreich dieses Argument nur eine untergeordnete Rolle im politischen Diskurs spielt (Marterbauer und Schürz 2007), sodass die Hinweise aus dem Interview in dem Online-Forum nicht aufgenommen wurden.

In Form der Vernachlässigung der Problematik der Unternehmensnachfolge konnte die von uns vorgenommene Untersuchung einen wesentlichen Unterschied zu jenen Expertendiskursen feststellen, auf die sich die Analyse dominanter Begründungsstrukturen bei Gegnern und Befürwortern der Steuer bislang beschränkt hat (vgl. Beckert 2004). In Übereinstimmung mit der inhaltsanalytischen Auswertung von Parlamentsdebatten zeigt sich dagegen, wie sowohl Gegner als auch Befürworter der Erbschaftssteuer bestimmte allgemeine normative Prinzipien als Bezugspunkte nutzen, aus diesen Prinzipien jedoch gegenteilige Schlüsse ziehen. Dies gilt hier insbesondere für das Leistungsprinzip, das einerseits als Argument für den Schutz des vom Erblasser selbst erwirtschafteten Vermögens angeführt wird, andererseits 
als Begründung für die Besteuerung der sich leistungslos bereichernden Erben. Der Bezug auf Wertprinzipien fügt sich in die Annahmen der économie des conventions (Boltanski und Thévenot 2007; Diaz-Bone 2015; Storper und Salais 1997), wonach Individuen sich in der Rechtfertigung ihres Handelns auf abstrakte, gesellschaftlich legitimierte Rechtfertigungsordnungen beziehen.

Politisch lässt sich aus unseren Analysen der Schluss ziehen, dass die Befürworter der Steuer vermutlich erst dann eine Mehrheit bilden können, wenn es ihnen gelingt, die Gegner davon zu überzeugen, dass die Erträge der Erbschaftssteuer nicht einfach in die Hände ,inkompetenter" und ,,verschwenderischer" staatlicher Akteure gelangen, sondern einen sinnvollen Beitrag zur Gestaltung des Gemeinwesens leisten. Dies könnte möglicherweise erreicht werden, indem die Erbschaftssteuer in der politischen Auseinandersetzung gezielt mit auf Chancengleichheit zielenden Investitionen - etwa im Bildungsbereich - verknüpft wird.

Weitere Forschung zu den einzelnen Argumenten und Bestandteilen des Diskurses um die Erbschaftssteuer wäre im Zusammenhang mit der Untersuchung der Legitimation sozialer Ungleichheit sinnvoll (Sachweh 2010). Darüber hinaus könnte die Untersuchung auf weitere Länder ausgedehnt und so eruiert werden, wie die Begründung von Haltungen zur Erbschaftssteuer - und damit zum gesellschaftlichen Umgang mit Vermögensunterschieden - in den jeweiligen nationalen Kontexten variiert.

Danksagung Für die Mitarbeit bei der Durchführung der Inhaltsanalyse danken wir Moritz Höfeld. Für hilfreiche Kommentare zu früheren Versionen des Artikels bedanken wir uns bei Philipp Korom, Lothar Krempel, Mark Lutter, Benjamin C. Seyd sowie den Reviewern des BJS.

\section{Literatur}

Altzinger, W., \& Humer, S. (2013). Simulation des Aufkommens verschiedener Erbschaftsbesteuerungen. Wien: Wirtschaftsuniversität Wien.

Backhaus, K., Erichson, B., Plinke, W., \& Weiber, R. (2016). Multivariate Analysemethoden. Eine anwendungsorientierte Einführung. Berlin: Springer.

Beckert, J. (2004). Unverdientes Vermögen: Soziologie des Erbrechts. Frankfurt a. M.: Campus.

Beckert, J. (2013). Erben in der Leistungsgesellschaft. Frankfurt a. M.: Campus.

Beckert, J., \& Arndt, L. R. (2016). Unverdientes Vermögen oder illegitimer Eingriff in das Eigentumsrecht? Der öffentliche Diskurs um die Erbschaftssteuer in Deutschland und Österreich. MPIfG Discussion Paper Nr. 16/8. http://www.mpifg.de/pu/mpifg_dp/dp16-8.pdf.

Best, S. J., \& Krueger, B. S. (2005). Analyzing the representativeness of internet political participation. Political Behavior, 27, 183-216.

Blom, R., Carpenter, S., Bowe, B. J., \& Lange, R. (2014). Frequent contributors within US newspaper comment forums. An examination of their civility and information value. American Behavioral Scientist, 58, 1314-1328.

Boltanski, L., \& Thévenot, L. (2007). Über die Rechtfertigung: Eine Soziologie der kritischen Urteilskraft. Hamburg: Hamburger Edition.

Bruckner, R. (2014). „Erbe und Arbeit gleich besteuern“. Interview mit Jens Beckert. Der Standard, 13.12.2014. http://derstandard.at/2000008561989/Erbe-und-Arbeit-gleich-besteuern. Zugegriffen: Februar 2016. (Basisartikel der analysierten Diskussionen)

Diaz-Bone, R. (2015). Die „Economie des conventions “: Grundlagen und Entwicklungen der neuen französischen Wirtschaftssoziologie. Wiesbaden: Springer VS.

Escher, T. (2013). Wi(e)der die ,üblichen Verdächtigen“"? Politische Beteiligung via Internet. In J. Wolling, M. Seifert \& M. Emmer (Hrsg.), Politik 2.0?: Die Wirkung computervermittelter Kommunikation auf den politischen Prozess (S. 131-151). Baden-Baden: Nomos.

Friedrichs, J. (2015). Wir Erben. Was Geld mit Menschen macht. Berlin: Berlin Verlag. 
Fuchs, C. (2006). eParticipation research: A case study on political online debate in Austria. http:// citeseerx.ist.psu.edu/viewdoc/download?doi=10.1.1.473.7117\&rep=rep1\&type=pdf. Zugegriffen: Oktober 2016.

Gaisbauer, H., Neumaier, O., Schweiger, G., \& Sedmak, C. (Hrsg.). (2013). Erbschaftssteuer im Kontext. Wiesbaden: Springer VS.

Haas, A., Keyling, T., \& Brosius, H.-B. (2010). Online-Diskussionsforen als Indikator für interpersonale (Offline-)Kommunikation? Methodische Ansätze und Probleme. In N. Jackob, T. Zerback, O. Jandura \& M. Maurer (Hrsg.), Das Internet als Forschungsinstrument und -gegenstand in der Kommunikationswissenschaft (S. 246-266). Köln: Herbert von Halem.

Harris, B. D., Morgan, C. V., \& Gibbs, B. G. (2014). Evidence of political moderation over time: Utah's immigration debate online. New Media \& Society, 16, 1309-1331.

Hucht, M. (2015). Vermögensverteilung in Deutschland: „Viele Erben fühlen sich schuldig“. Interview mit Julia Friedrichs. Spiegel Online, 18.3.2015. http://www.spiegel.de/wirtschaft/soziales/erbschaft-undvermoegensteuer-julia-friedrich-ueber-die-last-mit-dem-erbe-a-1023477.html. Zugegriffen: Februar 2016. (Basisartikel der analysierten Diskussionen)

Infratest dimap (2013). ARD-DeutschlandTREND Mai 2013. http://www.infratest-dimap.de/fileadmin/_ migrated/content_uploads/dt1305_bericht_01.pdf. Zugegriffen: Oktober 2016.

Kaiser, H. F. (1974). An index of factorial simplicity. Psychometrika, 39, 31-36.

Market Institut (2014). Erbschafts- und Vermögenssteuer. Brauchen wir diese Steuern? www.market.at/de/ market-aktuell/news/entity.detail/action.view/key.1117.html. Zugegriffen: Oktober 2016.

Marterbauer, M., \& Schürz, M. (2007). Der Streit um die Abschaffung der Erbschaftssteuer in Österreich. WISO: Wirtschafts- und sozialpolitische Zeitschrift, 30, 35-52.

Prabhakar, R. (2012). What do the public think of taxation? Evidence from a focus group study in England. Journal of European Social Policy, 22, 77-89.

Sachweh, P. (2010). Deutungsmuster sozialer Ungleichheit. Frankfurt a. M.: Campus.

Schmidt, M. (2015). Sieben von zehn Deutschen finden Erbschaftssteuer unfair. YouGov, 3.4.2015. https://yougov.de/news/2015/04/03/sieben-von-zehn-deutschen-finden-erbschaftssteuer-/. Zugegriffen: Oktober 2016.

Schweiger, W., \& Weihermüller, M. (2008). Öffentliche Meinung als Online-Diskurs. Ein neuer empirischer Zugang. Publizistik, 53, 535-559.

Stark, J., Kogler, C., Gaisbauer, H., Sedmak, C. \& Kirchler, E. (2016). Differentiating views of inheritance: The free association task as a method to assess social representations of wealth, inherit, and bequeath. Review of Behavioral Economics, 3, 91-111.

Storper, M., \& Salais, R. (1997): Worlds of production: The action frameworks of the economy. Cambridge: Harvard University Press.

Strandberg, K. (2008). Public deliberation goes on-line? An analysis of citizens' political discussions on the internet prior to the finnish parliamentary elections in 2007. Javnost - the Public, 15, 71-89.

Tiefensee, A., \& Grabka, M. M. (2017). Das Erbvolumen in Deutschland dürfte um gut ein Viertel größer sein als bisher angenommen. DIW Wochenbericht Nr. 27. Berlin: Deutsches Institut für Wirtschaftsforschung.

Weber, L. M., Loumakis, A., \& Bergman, J. (2003). Who participates and why? An analysis of citizens on the internet and the mass public. Social Science Computer Review, 21, 26-42.

Jens Beckert geb. 1967. Prof. Dr., Direktor am Max-Planck-Institut für Gesellschaftsforschung in Köln. Forschungsschwerpunkte: Wirtschaftssoziologie, Soziologie der Erbschaft, soziologische Theorie.

H. Lukas R. Arndt geb. 1989. MSc, Forschungsassistent am Max-Planck-Institut für Gesellschaftsforschung in Köln. Forschungsschwerpunkte: Wirtschaftssoziologie, Politische Soziologie, soziale Ungleichheit. 